\title{
Mesenchymal stem cell effects on T-cell effector pathways
}

\author{
Michelle M Duffy, Thomas Ritter, Rhodri Ceredig and Matthew D Griffin*
}

\begin{abstract}
Mesenchymal stem (stromal) cells (MSCs) are rare, multipotent progenitor cells that can be isolated and expanded from bone marrow and other tissues. Strikingly, MSCs modulate the functions of immune cells, including T cells, B cells, natural killer cells, monocyte/macrophages, dendritic cells, and neutrophils. T cells, activated to perform a range of different effector functions, are the primary mediators of many autoimmune and inflammatory diseases as well as of transplant rejection and graft-versus-host disease. Well-defined T-cell effector phenotypes include the CD4+ (T helper cell) subsets Th1, Th2, and Th17 cells and cytotoxic T lymphocytes derived from antigen-specific activation of naïve $C D 8^{+}$precursors. In addition, naturally occurring and induced regulatory $T$ cells $\left(T_{\text {reg }}\right)$ represent $C D 4^{+}$and $C D 8^{+} T$-cell phenotypes that potently suppress effector $T$ cells to prevent autoimmunity, maintain self-tolerance, and limit inflammatory tissue injury. Many immune-mediated diseases entail an imbalance between $T_{\text {reg }}$ and effector T cells of one or more phenotypes. MSCs broadly suppress T-cell activation and proliferation in vitro via a plethora of soluble and cell contact-dependent mediators. These mediators may act directly upon T cells or indirectly via modulation of antigen-presenting cells and other accessory cells. MSC administration has also been shown to be variably associated with beneficial effects in autoimmune and transplant models as well as in several human clinical trials. In a small number of studies, however, MSC administration has been found to aggravate T cell-mediated tissue injury. The multiple effects of MSCs on cellular immunity may reflect their diverse influences on the different T-cell effector subpopulations and their capacity to specifically protect or induce $T_{\text {req }}$ populations. In this review, we focus on findings from the recent literature in which specific modulatory effects of MSCS on one or more individual effector T-cell subsets and $T_{\text {reg }}$ phenotypes have been examined in vitro, in relevant animal models of in vivo immunological disease, and in human subjects. We conclude that MSCs have the potential to directly or indirectly inhibit diseaseassociated Th1, Th2, and Th17 cells as well as cytotoxic T lymphocytes but that many key questions regarding the potency, specificity, mechanistic basis, and predictable therapeutic value of these modulatory effects remain unanswered.
\end{abstract}

\section{An introduction to mesenchymal stem cell modulation of $\mathrm{T}$ cell-mediated immune responses}

$\mathrm{T}$ lymphocytes ( $\mathrm{T}$ cells) are the primary cellular effectors of the adaptive immune system and their functional properties are central to antigen specificity and memory associated with cognate immunity [1-3]. Antigen-specific activation and differentiation of naïve $\mathrm{T}$ cells result in the generation of a range of $\mathrm{T}$-cell phenotypes that may be defined by the acquisition of characteristic cytokine

\footnotetext{
*Correspondence: matthew.griffin@nuigalway.ie

Regenerative Medicine Institute (REMEDI), National Centre for Biomedical Engineering Science (NCBES) and School of Medicine, Nursing and Health Sciences, Orbsen Building, National University of Ireland Galway, University Road, Galway, Ireland
}

secretion profiles, cytolytic mechanisms, or counterregulatory properties [1-3]. In the wake of antigenspecific adaptive immune responses, a small proportion of activated $\mathrm{T}$ cells persist as memory cells and have the capacity to respond more rapidly and potently to secondary encounters with the same antigen [1,3]. These memory cells may retain the effector phenotype imprinted upon them during primary activation [1]. When these memory cells are appropriately coordinated and regulated, the diversity of T-cell effector phenotypes allows immune protection against a multitude of pathogenic microorganisms while maintaining self-tolerance and homeostasis [2]. On the other hand, overexuberant pro-inflammatory $\mathrm{T}$-cell responses may lead to autoimmune and allergic diseases, including multiple sclerosis, inflammatory bowel disease, type 1 diabetes mellitus, and asthma [4-7]. Furthermore, life-saving treatments such as 
allogeneic bone marrow (BM) and solid organ transplantation may be complicated by alloantigen-specific T-cell immune responses, resulting in graft-versus-host disease (GvHD) or transplant rejection [8].

Mesenchymal stem (or stromal) cells (MSCs) are a heterogeneous population of fibroblast-like progenitor cells that may be isolated and expanded from BM, umbilical cord, fat, gingiva, and other tissues [9]. They have the capacity to self-renew and differentiate into various mesodermal cell lineages, including adipocytes, osteocytes, and chondrocytes under controlled culture conditions [9]. In the past two decades, MSCs have garnered considerable attention for their potential use as regenerative therapeutic agents in a range of acute and chronic diseases [8-11]. Mechanistically, the beneficial effects of MSC therapies have been more frequently linked to their 'trophic' (paracrine) effects rather than their ability to transdifferentiate [11]. Specifically, MSCs are now viewed as having potent anti-inflammatory and immune-modulating properties that, in many studies, have been shown to be associated with inhibition of effector T-cell activation with or without a concomitant increase in regulatory $\mathrm{T}$ cell $\left(\mathrm{T}_{\text {reg }}\right)$ numbers $[4,6,10-12]$. The T-cell suppressive effects of MSCs were initially described over a decade ago [13] and have since been reported consistently for both $\mathrm{CD} 4^{+} \mathrm{T}$ helper (Th) cells and $\mathrm{CD}^{+}$cytotoxic $\mathrm{T}$ lymphocytes (CTLs) $[8,11,14]$. Suppression of $\mathrm{T}$ cells by MSCs may be direct or may occur indirectly via modulatory effects on antigenpresenting cells such as dendritic cells (DCs), resulting in altered cytokine expression and impaired antigen presentation [15-17]. MSCs themselves demonstrate a lack of stimulatory capacity toward T cells $[18,19]$. MSCs isolated from various sources (BM, adipose tissue, and Wharton's jelly) have been reported to equally suppress proliferation of $\mathrm{CD}^{+}$and $\mathrm{CD} 8^{+} \mathrm{T}$-cell subsets in a dosedependent fashion [20]. Reported roles for both cell-cell contact and release of soluble factors in MSC-mediated T-cell suppression are evident throughout the literature, and numerous candidate mediators have been reported: prostaglandin $\mathrm{E}_{2}\left(\mathrm{PGE}_{2}\right)$, indoleamine-2,3-dioxygenase, nitric oxide, interleukin (IL)-27, transforming growth factor-beta (TGF- $\beta$ ), monocyte chemotactic protein 1 (MCP-1/CCL2), human leukocyte antigen G, and intracellular adhesion molecule 1 among others [8,10-12,17, 21-24]. The abundance of mediators identified to date suggests that MSCs exploit different immunosuppressive mechanisms under different disease conditions. MSC therapy has been successful in a range of disease models and some clinical conditions known to be associated with damaging effector $\mathrm{T}$-cell responses or failure of $\mathrm{T}_{\text {reag }}$ mediated counter-regulation or both $[4,6,8,11,22,25-28]$. Overall, it is now very well established that MSCs exert diverse and potent modulatory effects on the T-cell compartment of the immune system, most of which are suppressive in nature and of potential therapeutic value. Nevertheless, some significant controversies and a basic lack of information regarding the range of effects that MSCs have on individual T-cell effector subsets remain. In the remaining sections of this review, we focus on the most recent data related to MSC modulation of individual well-defined Th cell and CTL effector phenotypes in vitro and in vivo. Where possible, we emphasize the relevance of current knowledge on this topic to diseases for which MSC therapy is perceived to be beneficial. We also highlight key gaps in our understanding and important unanswered questions that may be the subject of future studies.

\section{Mesenchymal stem cell modulation of T helper cell subsets}

Th cells are cytokine-producing $\mathrm{CD} 4^{+}$cells that recognize peptides presented to them by major histocompatibility complex (MHC) class II molecules [1-3]. Differentiation of Th cells into effector cells depends largely on the cytokine milieu present at the time of antigen presentation and activation [1-3]. In the context of this article, we will focus on reviewing recent progress (summarized in Figure 1) in understanding MSC effects on the welldescribed subsets Th1, Th2, and Th17 cells, $\mathrm{T}_{\text {reg }}$, and CTLs with emphasis, where possible, on mechanistic and disease-specific in vivo studies.

\section{Thelper type 1 cells}

Th1 cell induction occurs when $\mathrm{CD} 4^{+} \mathrm{T}$ cells are activated in the presence of IL-12, interferon-gamma (IFN- $\gamma$ ), and IL-27 [29]. IFN- $\gamma$ is the characteristic cytokine produced by Th1 cells in addition to tumor necrosis factor (TNF). Known effector functions of Th1 cells include activation and recruitment of macrophages to sites of inflammation and induction of immunoglobulin (Ig) G2a production by $B$ cells [2]. Th1 cells are responsible for the clearance of intracellular pathogens and delayed-type hypersensitivity (DTH) reactions by amplifying cellular immunity [29]. DTH reactions are mediated by both Th1 cells and CTLs [25], and, through their role as coordinators of this form of immune response, Th1 cells have the capacity to cause maladaptive tissue damage. Examples of Th1 cell-mediated inflammatory and autoimmune diseases are type 1 diabetes mellitus and Crohn's disease [7]. The literature to date indicates that MSCs exert primarily suppressive effects on Th1 cell differentiation and effector function, and evidence favors predominantly indirect mechanisms. In vitro, the generation of Th1 cells is reduced in mixed lymphocyte cultures containing MSCs or MSC-conditioned medium, likely due to inhibition of Th1 cellstimulating properties of DCs [30]. In the in vivo setting, Lim and colleagues [25] recently demonstrated that 


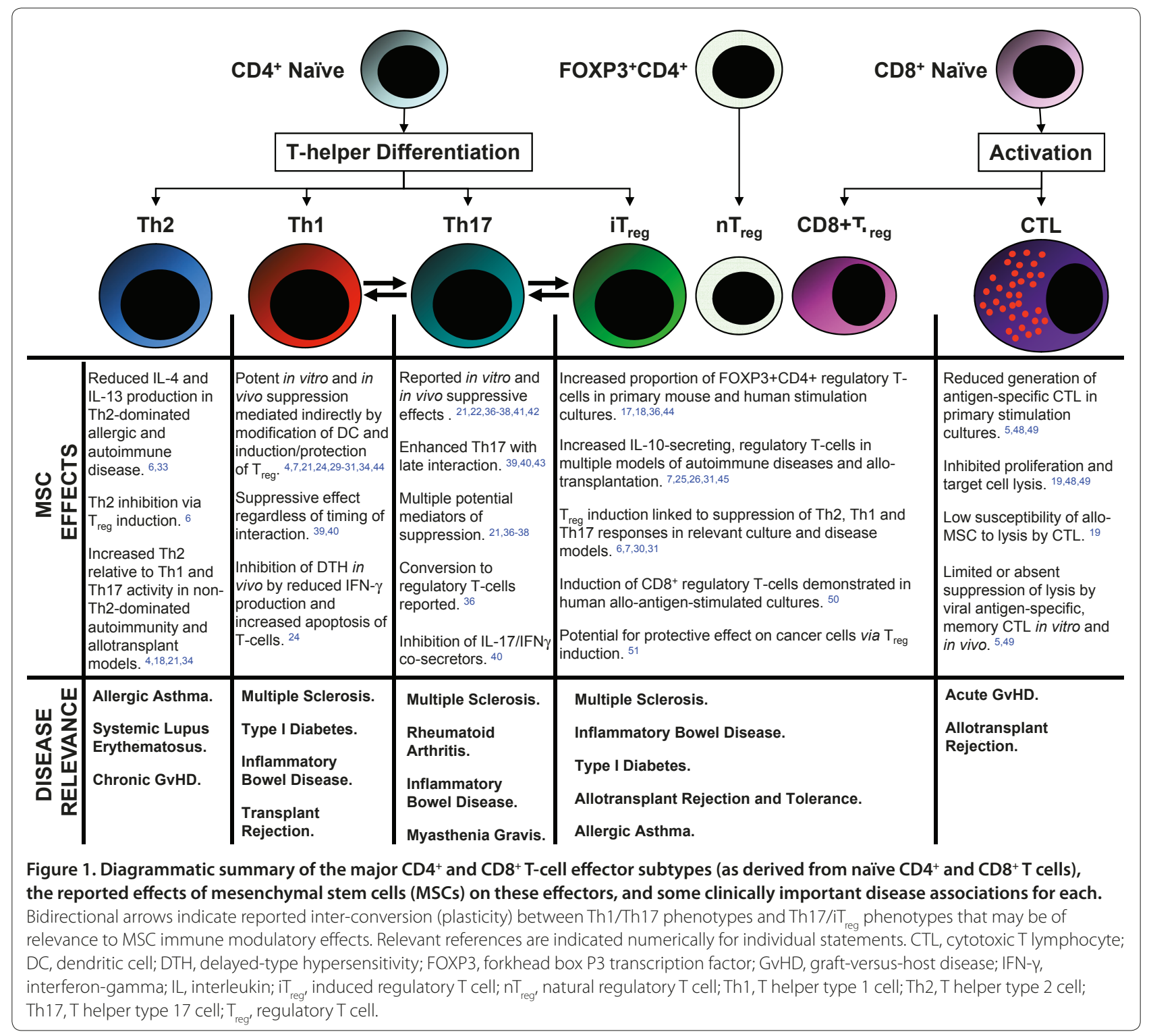

infusion of MSCs attenuated cutaneous DTH in mice and that this effect was associated with reduced infiltration of $\mathrm{CD}^{+}$and $\mathrm{CD} 8^{+} \mathrm{T}$ cells at the challenge site and increased apoptosis of activated $\mathrm{T}$ cells in the draining lymph nodes. MSCs were detected close to the germinal center and paracortical region in lymph nodes [25], suggesting that they modulate immune responses directly in the area where DCs are likely to activate T cells. In experimental colitis (an animal model of inflammatory bowel disease), dose-dependent xenogenic, allogenic, and autologous adipose-derived MSCs ameliorated disease activity and were specifically associated with reduced IFN- $\gamma$-producing Th1 cells in association with increased numbers of forkhead box P3 (FOXP3)-expressing $\mathrm{T}$ cells $\left(\mathrm{T}_{\mathrm{reg}}\right)$ [7]. Furthermore, when treated with either total $\mathrm{CD} 4^{+}{ }^{+} \mathrm{T}$ cells or $\mathrm{T}_{\text {reg }}$-depleted $\mathrm{CD}^{+} \mathrm{T}$ cells from mesenteric lymph nodes of MSC-treated colitic mice, mice with induced colitis demonstrated attenuated and enhanced colitis, respectively [7]. These data suggest that the administration of MSCs in experimental colitis dampens Th1 cell responses via induction of $T_{\text {reg }}$ but does not eliminate Th1 cells entirely. MSCs have also been used experimentally to effectively prevent or treat Th1 cell-mediated autoimmune diabetes mellitus in streptozotocin-treated rats and in nonobese diabetic (NOD) mice [31,32]. In the rat model, the protective effects observed following administration of MSCs were shown to be associated with increased IL-10 and IL-13 expression by T cells and with increased frequencies of both $\mathrm{CD}^{+}$and $\mathrm{CD}^{+} \mathrm{FOXP3}^{+}$ $\mathrm{T}$ cells as opposed to a direct reduction of IFN- $\gamma$ producing $\mathrm{T}$ cells [31]. In NOD mice, a single MSC injection minimized beta-cell destruction following 
transfer of diabetogenic T cells. Protection was shown to be associated with MSC migration to pancreatic lymph nodes and with induction of IL-10-producing FOXP3 ${ }^{+}$ $\mathrm{T}_{\text {reg }}$ [32]. These examples from the recent literature indicate that, in clinically relevant disease settings, MSCs consistently suppress harmful autoimmune Th1 cell responses by predominantly indirect mechanisms, including modulation of antigen-presenting DCs and promotion of naturally occurring or induced FOXP3expressing $\mathrm{T}_{\mathrm{reg}}$.

\section{T helper type 2 cells}

Th2 cell differentiation occurs when $\mathrm{CD}^{+} \mathrm{T}$ cells are activated in the presence of IL-4, which itself is produced by Th2 cells in addition to IL-5, IL-9, IL-10, and IL-13 $[2,3,29]$. The role of Th2 cells in adaptive immunity is linked to host defense against extracellular parasites, to antibody class switching to IgG1 and IgE in B cells, and to recruitment of eosinophils $[2,3,29,33]$. Dysregulated Th2 cell responses are associated with allergic diseases such as asthma [2]. Very few studies have examined MSC effects on immune-mediated diseases in which Th2 cell responses are dominant. However, Kavanagh and Mahon [6] recently reported that allogeneic MSC administration reduced the number of infiltrating eosinophils, suppressed IgE induction, and inhibited IL-13 and IL-4 production in a mouse model of ovalbumin-induced airway inflammation. Additionally, increases in IL-10 and FOXP3 expression were observed in this study, suggesting that MSCs suppress allergen-specific Th2 cell responses in allergic airway inflammation in part via induction of $\mathrm{T}_{\mathrm{reg}}$. As further evidence of this, depletion of $\mathrm{T}_{\text {reg }}$ resulted in reversal of the protective effects of the MSCs [6]. In human subjects with chronic GvHD (which is also characterized by predominant Th2 cell activity), MSC infusion has been reported to result in clinical improvement with a reduction in IL-4- and IL-10-producing $\mathrm{T}$ cells and a concomitant increase in IL-2- and IFN- $\gamma$ producing cells [34].

In other circumstances, there is evidence that MSCs may favor the emergence of Th2 phenotype $\mathrm{T}$ cells. Bai and colleagues [4] demonstrated, for example, that mice treated with human BM-derived MSCs recovered function from limb paralysis in relapsing-remitting and chronic experimental allergic encephalomyelitis (EAE), a model for multiple sclerosis, by induction of Th2 cells. In this study, neurological improvement was associated with reduced $\mathrm{CD}_{4} 5^{+}$leukocytic infiltration of the brain and spinal cord, with increased levels of the Th2 cell-related cytokines IL-4 and IL-5, and with potent reduction in the Th1/Th17 cell-related cytokines IL-17, IFN- $\gamma$, TNF, and IL-12 [4]. The results suggested that MSC administration in EAE favorably altered the balance between proinflammatory Th1/Th17 cell and anti-inflammatory Th2 cell responses. Likewise, Fiorina and colleagues [35] reported a shift in Th1/Th2 cell balance toward Th2 cells following allogeneic MSC administration to NOD mice. Further evidence supporting a shift toward Th2 cell responses is provided by Batten and colleagues [18], who describe the use of human BM-derived MSCs for tissue engineering of a heart valve. $\mathrm{CD}^{+} \mathrm{T}$ cells co-cultured with MSCs expressed lower levels of IL-1- $\alpha$ and $-\beta$, TNF, and IFN- $\gamma$ but higher levels of IL-5, IL-8, and IL-3 in response to allogeneic peripheral blood mononuclear cells. Consistent with the findings of Kavanagh and Mahon [6], those of Batten and colleagues [18] additionally indicated increased expression of FOXP3 in $\mathrm{CD}^{+} \mathrm{T}$ cells cocultured with MSCs, suggesting the induction of a $\mathrm{T}_{\text {reg }}$ phenotype. Thus, though relatively limited, the experimental evidence to date suggests that MSCs suppress effector function of Th2 cells in Th2 cell-predominant inflammation. In other $\mathrm{T}$ cell-mediated immunological disorders, however, predominant MSC suppression of the Th1 and Th17 cell pathways may result in a relative skewing toward less damaging $\mathrm{Th} 2$ and $\mathrm{T}_{\text {reg }}$ phenotypes. Whether MSCs actively induce the differentiation and expansion of Th2 cells during primary or secondary antigen-specific immune responses has not been well tested but appears less likely.

\section{Thelper type 17 cells}

The Th17 cell effector phenotype is defined by preferential secretion of IL-17A (IL-17) along with other cytokines, including IL-17F, IL-21, and IL-22. Th17 cells are pro-inflammatory and protect against extracellular pathogens, including fungi, mycobacteria, and Gram-negative bacteria, via recruitment of neutrophils [36]. Th17 cells may also be pathogenic and have been shown to have an important role in immunological diseases, including rheumatoid arthritis, multiple sclerosis, and inflammatory bowel disease [36]. TGF- $\beta$ and IL-6, with or without IL-21, IL-23, and IL-1, are necessary for the induction and expansion of Th17 cells from naïve CD4+ $4^{+}$precursors $[2,36]$. Recently, MSC effects on the Th17 cell differentiation pathway have been examined in mice and humans. Ghannam and colleagues [37] observed that human MSCs induce regulatory characteristics in Th17 cells in an inflammatory environment by downregulating the Th17 cell-specific transcription factor ROR $\gamma$ t (retinoidacid receptor-related orphan receptor gamma t) and upregulating FOXP3. Moreover, when re-purified, these regulatory-phenotype Th17 cells suppressed proliferation of newly initiated $\mathrm{CD}^{+} \mathrm{T}$ cells [37]. In vivo, MSC administration has been shown to suppress the development of EAE via a reduction in IL-17 production in the central nervous system along with reduced IFN- $\gamma$, TNF, and IL-23 and increased TGF- $\beta$ and IL-4 [22]. Inhibition of Th17 cell activity in EAE has also been 
reported by Zappia and colleagues [38] and Rafei and colleagues [39], although the studies to date have identified different mechanisms for the MSC anti-Th17 cell effect, including IL-27 [22], alternatively cleaved MCP-1 [39], and induction of a state of T-cell anergy [38]. In our own hands, MSCs potently suppress the in vitro differentiation and re-activation of mouse Th17 cells derived from naïve and memory precursors via cyclooxygenase 2 upregulation and $\mathrm{PGE}_{2}$ production (MM Duffy, R Ceredig, and MD Griffin, unpublished work).

Although these studies indicate that MSCs have the potential to suppress Th17 cell-mediated immunity and may do so by several mechanisms, some evidence for a Th17 cell-promoting effect of MSCs also exists. For example, Carrión and colleagues [40] observed that MSCs promoted Th17 cells while inhibiting Th1 cells in vitro if their addition to mouse T-cell differentiation cultures was delayed by 3 days. Similarly, Darlington and colleagues [41] observed that MSC-conditioned medium suppressed human Th1 cells in vitro while having an opposing effect on Th17 cells. In the same study, MSCconditioned medium was found to reduce numbers of IL-17/IFN- $\gamma$ double-expressing $\mathrm{CD} 4^{+} \mathrm{T}$ cells; this finding may have clinical implications for patients with multiple sclerosis as this subset was recently described in immune-mediated demyelinating disease [41]. Whether MSCs inhibit or enhance disease-associated Th17 cells in vivo is less well understood, although Ghannam and colleagues [37] observed that MSCs suppressed the production of IL-17 and IL-22 by established human Th17 cell clones with a paradoxical increase in IL-10producing cells. Furthermore, Rafei and colleagues [39] demonstrated amelioration of EAE and inhibition of Th17 cell activity when MSCs were first administered 1 week after the onset of neurological signs of disease, suggesting the inhibition of established T-cell effector responses. Inhibition of Th17 cell-mediated inflammation and autoimmunity by MSC administration has also been reported in models of type 1 diabetes mellitus, collageninduced arthritis, and experimental autoimmune myasthenia gravis in association with shifts toward increased Th2 or $\mathrm{T}_{\text {reg }}$ activity or both $[23,42,43]$. Overall, a significant amount of evidence for specific effects of MSCs on the Th17 cell effector pathway is emerging. These effects would appear to be suppressive under diverse conditions but with the potential to enhance Th17 cell activity under some circumstances. In this regard, it is worth noting that MSCs may act as a source of IL-6, which is one of the primary mediators of Th17 cell differentiation [44]. The role of MSC-produced IL- 6 is likely to be more complex, however, as MSCs derived from IL-6-deficient mice were less effective than wild-type MSCs in suppressing inflammation associated with collagen-induced arthritis in a study by Bouffi and colleagues [42].

\section{Regulatory $\mathrm{T}$ cells}

A subset of $\mathrm{CD}^{+} \mathrm{T}$ cells have been identified as having regulatory (suppressor) functions that are essential for the prevention of autoimmunity and the resolution of inflammatory processes. These $\mathrm{CD}^{+}{ }^{+} \mathrm{T}_{\text {reg }}$ are best characterized by surface expression of the IL-2 receptor alpha chain (CD25) and, more specifically, by intracellular expression of the transcription factor FOXP3. They can be further subdivided into naturally occurring $\mathrm{T}_{\text {reg }}\left(\mathrm{nT}_{\text {reg }}\right)$ that develop in the thymus or induced $\mathrm{T}_{\text {reg }}\left(\mathrm{iT}_{\text {reg }}\right)$ that differentiate from naïve peripheral $\mathrm{CD} 4^{+} \mathrm{T}$ cells in the presence of TGF- $\beta$ [2]. $\mathrm{T}_{\text {reg }}$ exert potent immunosuppressive effects via cell-cell contact and production of soluble factors and may negatively regulate the activation of each of the major Th cell subtypes as well as other immune and inflammatory cells [2]. As indicated at several points in the preceding sections, there has been a consistent theme among many in vitro and in vivo studies in support of MSC enhancement of $\mathrm{T}_{\text {reg }}$ number and activity $[8,10]$. English and colleagues [17] showed that human $\mathrm{FOXP}^{+} \mathrm{CD} 25^{\text {high }} \mathrm{T}_{\text {reg }}$ were induced upon coculture of allogeneic MSCs and CD4 ${ }^{+} \mathrm{T}$ cells and exerted suppressive activity when re-purified and added to a newly initiated mixed lymphocyte culture. This was corroborated in a study of human adipose tissue-derived MSCs that, in addition to reducing IL-17, TNF, and IFN- $\gamma$ production, induced IL-10-producing, $\mathrm{FOXP3}^{+} \mathrm{T}_{\mathrm{reg}}$ in vitro among collagen-specific peripheral blood $\mathrm{T}$ cells of patients with rheumatoid arthritis [45]. Upon reisolation, the $\mathrm{T}_{\text {reg }}$ originally generated in the presence of MSCs had the capacity to inhibit IFN- $\gamma$ production and proliferation of a subsequent collagen-stimulated T-cell culture [45]. In in vivo models of kidney, liver, and heart allotransplantation, several laboratories have linked the protective effects observed with MSC therapy directly to the presence of $\mathrm{T}_{\text {reg }}[26,27,46]$. The importance of the induced $\mathrm{T}_{\text {reg }}$ populations in such transplant models, as well as in some models of allergic and autoimmune disease, is well illustrated by the induction of graft rejection or loss of therapeutic benefit following $\mathrm{T}_{\text {reg }}$ depletion $[6,8,26]$. One concern that requires further investigation regarding $\mathrm{MSC}$-induced $\mathrm{T}_{\text {reg }}$ relates to the potential for phenotypic plasticity of pro- and antiinflammatory $\mathrm{CD} 4^{+} \mathrm{T}$-cell subsets under varying in vivo conditions. For example, as highlighted in a review by Afzali and colleagues [47], $\mathrm{T}_{\text {reg }}$ may be converted to a Th17 cell phenotype when exposed to inflammatory stimuli. In such circumstances, MSC-induced $\mathrm{T}_{\text {reg }}$ may exacerbate the disease state. Further human studies will be essential to fully elucidate the clinical relevance and robustness of MSC-induced $\mathrm{T}_{\text {reg }}$ in vivo, as evidenced by the study of Carrión and colleagues [48] in which the presence of MSC-induced $\mathrm{T}_{\text {reg }}$ did not alter the disease course in two patients with systemic lupus erythematosus. 


\section{Mesenchymal stem cell modulation of cytotoxic T lymphocytes}

CD8 ${ }^{+}$CTLs recognize cytosolic antigen-derived peptides presented by MHC class I and thus are essential for the destruction of virus-infected cells and tumor cells. Following their primary activation by professional antigen-presenting cells such as DCs, CTLs induce cell death upon secondary encounter of antigen expressed by any cell type via pro-apoptotic surface receptors or targeted release of cytotoxic granules [3,19]. CTLs also release IFN- $\gamma$, TNF, and lymphotoxin- $\alpha$ to inhibit viral replication and to recruit macrophages to the site of infection [3]. Death receptor ligands such as TRAIL (TNF-related apoptosis-inducing ligand) and Fas ligand are upregulated on activated CTLs. In patients receiving allogeneic BM or hematopoietic stem cell transplants, activated CTLs mediate GvHD, causing damage to liver, intestine, skin, and other tissues [19]. Therapeutically administered MSCs have the potential to reduce disease severity in GvHD and other immune-mediated diseases via direct effects on CTLs as well as through inhibition of Th cell responses, which are required for full activation of CTLs [3]. For allogeneic MSC administration, the beneficial effects may also be limited by alloantigen-specific CTL-mediated MSC lysis. In a study by Rasmusson and colleagues [19], however, MSCs were resistant to CTL lysis despite the expression of MHC class I on their surface. MSCs were also unable to induce pro-inflammatory cytokine production or CD25 upregulation by CTLs [19]. This same group and others have also demonstrated that MSCs inhibit the formation of CTLs in mixed lymphocyte cultures and prevent CTL-associated lysis of target cells if added during the primary stimulation phase $[49,50]$. In contrast, MSCs were unable to suppress activated CTLs at the cytotoxic effector phase [50]. Such findings were corroborated by Karlsson and colleagues [5], who showed that, while MSCs potently suppressed primary alloantigen-induced proliferation and IFN- $\gamma$ production by human peripheral blood leukocytes, they had no effect on cytomegalovirus (CMV)-induced proliferation or IFN- $\gamma$ production. Furthermore, MSCs were unable to suppress proliferation or cytolytic killing in established CMV- or Epstein-Barr virus-specific CTL lines [5]. These observations are of particular clinical relevance to the treatment of GvHD patients who are at high risk from the reactivation of viral infections. However, in this case, administration of MSCs was ineffective toward CMV-mediated $\mathrm{CD}^{+}{ }^{+} \mathrm{T}$-cell effector functions while potently suppressing alloantigen-induced responses [5].

Interestingly, some of the protective effects of MSCs in GvHD may also result from the generation of $\mathrm{CD}^{+} \mathrm{T}_{\mathrm{reg}}$, as demonstrated by Prevosto and colleagues [51]. It is proposed that $\mathrm{CD}^{+} \mathrm{T}_{\text {reg }}$ may amplify the immune modulatory effects of MSCs because, when re-purified from peripheral blood leukocytes/MSC co-cultures, these cells potently suppressed subsequent peripheral blood leukocyte proliferation in response to alloantigen and to the non-specific mitogen phytohaemagglutinin [51]. It is also important to note that MSC-associated immune modulation may have detrimental effects in the setting of cancer. In a recent study by Patel and colleagues [52], the addition of MSCs to co-cultures of breast cancer cells and peripheral blood leukocytes resulted in enhanced $\mathrm{T}_{\text {reg }}$ numbers and Th2 cell-related cytokines as well as inhibited proliferation and release of granzyme B by CTLs, all of which resulted in protection of cancer cells from immune-mediated lysis. In vivo studies to examine this phenomenon are essential to fully understand the complex interaction between MSCs, T cells, and cancer cells and to ensure that MSC administration is not associated with recurrence or rapid metastasis of cancer in some patient groups. Overall, the experimental and clinical evidence to date suggests that MSCs exert both direct and indirect suppressive effects on the generation of antigen-specific CTLs and may foster the emergence of $\mathrm{CD}^{+} \mathrm{T}_{\text {reg }}$ but do not significantly inhibit the immune surveillance functions of pre-existing $\mathrm{CD} 8^{+}$ memory $\mathrm{T}$ cells.

\section{Concluding remarks and future directions}

The immune suppressive and anti-inflammatory properties of MSCs are now very well established and clearly encompass potent modulatory influences on the generation and disease-associated activity of multiple T-cell effector phenotypes [8-12]. Preclinical models provide a strong impetus for translating MSC therapy to widespread clinical use for a range of common, T-cellmediated autoimmune diseases and for prevention or treatment of transplant complications such as rejection and GvHD $[8,10]$. Despite this, a critical review of our current understanding of these effects and of recent developments in MSC clinical trials [28] indicates that much remains to be learned at both the mechanistic and logistic levels. The dizzying array of potential MSCassociated mediators of T-cell suppression, the many differences between small-animal immunological models and human immune-mediated diseases, and the lack of uniformity in MSC culture and administration protocols suggest that a more focused experimental pipeline will be required for the therapeutic potential to be realized in the near future. Table 1 summarizes a number of key questions that, on the basis of the literature to date, we believe to be important for translational progress in this field. In particular, we would highlight the need to better understand conditions in which MSC administration has been found to be ineffective or even harmful during $\mathrm{T}$ cell-mediated disease. Zappia and colleagues [38] 
Table 1. Key unanswered or incompletely answered questions about mesenchymal stem cell effects on T-cell effector functions

1 Is there a true hierarchy of suppressive potency for MSC effects on the three major Thelper cell effector pathways (for example, Th1 cells > Th17 cells > Th2 cells)?

2 Are individual T-cell effector phenotypes susceptible to different MSC mechanisms of suppression, or does suppressive mechanism vary by MSC culture condition or by disease pathogenesis (or by both)?

3 How important is cell-cell contact for the in vivo direct and indirect suppression by MSCs of harmful T-cell effector functions?

4 Is the apparent shift from Th1/Th17 to Th2/T responses following MSC therapy in some diseases due to true induction and expansion of antiinflammatory T-cell effector phenotypes or to their preferential survival?

5 What are the relative effects of MSCs on memory and pre-activated T-cell effectors compared with naïve T cells undergoing primary activation?

6 Do immunosuppressive potency and mechanism of action vary among the individual cells within heterogenous MSC cultures and can they be enhanced by MSC cloning or modification?

7 To what extent and under what circumstances are MSCs capable of promoting harmful T-cell effector functions?

8 How long-lasting is MSC modulation of antigen-specific T-cell effectors following single or multiple in vivo administration?

9 How can MSC in vivo immune suppressive potency, mechanism of action, and site of migration be better predicted for a given culture preparation prior to therapeutic administration?

10 Does the in vivo immunogenicity of allogeneic MSCs limit their long-term therapeutic benefit in autoimmune disease and allotransplantation?

11 What are the combined in vivo effects of MSC and other anti-T-cell therapies?

MSC, mesenchymal stem cell; Th1,T helper type $1 ;$ Th2, T helper type $2 ; \mathrm{Th} 17, \mathrm{~T}$ helper type $17 ; \mathrm{T}_{\text {reg' }}$, regulatory $\mathrm{T}$ cell.

demonstrated, for example, that the time of administration of MSCs was a critical parameter for successful treatment of EAE since MSC administration prior to or during the early disease course was effective whereas MSC benefit was lost once central nervous system inflammation was fully established. Similarly, in experimental arthritis, the timing of MSC administration, the relative effects of MSCs on different Th cell subsets, and the local joint conditions have been reported to critically determine the balance between therapeutic efficacy, lack of benefit, and detrimental effects $[40,42,44,53]$. These studies should in no way dampen enthusiasm for further preclinical and clinical applications of MSCs in disease conditions in which one or more T-cell effector pathways are known to be the primary cause for acute or chronic tissue damage. Rather, they highlight the complexity of the interactions that occur between stromal cells and cells of the immune system and the wealth of basic and therapeutic insights that can be gained from continued investigation of these interactions.

This article is part of a review series on Immunology and stem cells, edited by Christian Jorgensen. Other articles in the series can be found online at http://stemcellres.com/series/immunology

\section{Abbreviations}

BM, bone marrow; CMV, cytomegalovirus; CTL, cytotoxic T lymphocyte; DC, dendritic cell; DTH, delayed-type hypersensitivity; EAE, experimental allergic encephalomyelitis; FOXP3, forkhead box P3; GVHD, graft-versus-host disease; IFN-y, interferon-gamma; Ig, immunoglobulin; IL, interleukin; MCP-1, monocyte chemotactic protein 1; MHC, major histocompatibility complex; MSC, mesenchymal stem (stromal) cell; NOD, nonobese diabetic; PGE, prostaglandin $\mathrm{E}_{2^{\prime}}$ TGF- $\beta$, transforming growth factor-beta; Th, T helper; Th1, T helper type 1;Th2, Thelper type 2; Th17, T helper type 17; TNF, tumor necrosis factor; $T_{\text {reg' }}$ regulatory $T$ cell(s).

\section{Competing interests}

The authors declare that they have no competing interests.

\section{Acknowledgments}

The authors are funded by grants from Science Foundation Ireland (grant numbers SFI PI 06/IN.1/B652 to MMD and MDG, SFI PI 07/IN.1/B925 to TR, and SFI SRC 09/SRC/B1794 to MDG, TR, and RC), by a Science Foundation Ireland Stokes Professorship to RC, and by grants from the Health Research Board of Ireland (grant numbers HRA_HSR/2010/63 to MDG and RP/2007/60 to TR).

Published: 11 August 2011

\section{References}

1. Kaech SM, Wherry EJ, Ahmed R: Effector and memory T-cell differentiation: implications for vaccine development. Nat Rev Immuno/ 2002, 2:251-262.

2. Wan YY, Flavell RA: How diverse--CD4 effector T cells and their functions. J Mol Cell Biol 2009, 1:20-36.

3. Janeway C: T-cell mediated immunity. In Janeway's Immunobiology. 7th edition. Edited by Murphy K, Travers P, Walport M. New York: Garland Science, Taylor \& Francis Group; 2008:364-368.

4. Bai L, Lennon DP, Eaton V, Maier K, Caplan Al, Miller SD, Miller RH: Human bone marrow-derived mesenchymal stem cells induce Th2-polarized immune response and promote endogenous repair in animal models of multiple sclerosis. Glia 2009, 57:1192-1203.

5. Karlsson H, Samarasinghe S, Ball LM, Sundberg B, Lankester AC, Dazzi F, Uzunel M, Rao K, Veys P, Le Blanc K, Ringdén O, Amrolia PJ: Mesenchymal stem cells exert differential effects on alloantigen and virus-specific T-cell responses. Blood 2008, 112:532-541.

6. Kavanagh $\mathrm{H}$, Mahon BP: Allogeneic mesenchymal stem cells prevent allergic airway inflammation by inducing murine regulatory T cells. Allergy 2011, 66:523-531.

7. Gonzalez MA, Gonzalez-Rey E, Rico L, Buscher D, Delgado M: Adiposederived mesenchymal stem cells alleviate experimental colitis by inhibiting inflammatory and autoimmune responses. Gastroenterology 2009, 136:978-989.

8. English K, French A, Wood KJ: Mesenchymal stromal cells: facilitators of successful transplantation? Cell Stem Cell 2010, 7:431-442.

9. Barry FP, Murphy JM: Mesenchymal stem cells: clinical applications and biological characterization. Int J Biochem Cell Biol 2004, 36:568-584.

10. Griffin MD, Ritter T, Mahon BP: Immunological aspects of allogeneic 
mesenchymal stem cell therapies. Hum Gene Ther 2010, 21:1641-1655.

11. Caplan Al: Why are MSCs therapeutic? New data: new insight. J Pathol 2009, 217:318-324.

12. Barry FP, Murphy JM, English K, Mahon BP: Immunogenicity of adult mesenchymal stem cells: lessons from the fetal allograft. Stem Cells Dev 2005, 14:252-265.

13. Bartholomew A, Sturgeon C, Siatskas M, Ferrer K, McIntosh K, Patil S, Hardy W, Devine S, Ucker D, Deans R, Moseley A, Hoffman R: Mesenchymal stem cells suppress lymphocyte proliferation in vitro and prolong skin graft survival in vivo. Exp Hematol 2002, 30:42-48.

14. Gieseke F, Bohringer J, Bussolari R, Dominici M, Handgretinger R, Muller I: Human multipotent mesenchymal stromal cells use galectin-1 to inhibit immune effector cells. Blood 2010, 116:3770-3779.

15. Cutler AJ, Limbani V, Girdlestone J, Navarrete CV: Umbilical cord-derived mesenchymal stromal cells modulate monocyte function to suppress T cell proliferation. J Immunol 2010, 185:6617-6623.

16. Wang Q, Sun B, Wang D, Ji Y, Kong Q, Wang G, Wang J, Zhao W, Jin L, Li H: Murine bone marrow mesenchymal stem cells cause mature dendritic cells to promote T-cell tolerance. Scand J Immunol 2008, 68:607-615.

17. English K, Ryan JM, Tobin L, Murphy MJ, Barry FP, Mahon BP: Cell contact, prostaglandin $\mathrm{E}(2)$ and transforming growth factor beta 1 play nonredundant roles in human mesenchymal stem cell induction of CD4+CD25(High) forkhead box P3+ regulatory T cells. Clin Exp Immunol 2009, 156:149-160.

18. Batten P, Sarathchandra P, Antoniw JW, Tay SS, Lowdell MW, Taylor PM, Yacoub $\mathrm{MH}$ : Human mesenchymal stem cells induce T cell anergy and downregulate $\mathrm{T}$ cell allo-responses via the $\mathrm{TH} 2$ pathway: relevance to tissue engineering human heart valves. Tissue Eng 2006, 12:2263-2273.

19. Rasmusson I, Uhlin M, Le Blanc K, Levitsky V: Mesenchymal stem cells fail to trigger effector functions of cytotoxic T lymphocytes. J Leukoc Biol 2007, 82:887-893.

20. Najar M, Raicevic G, Boufker HI, Fayyad Kazan H, De Bruyn C, Meuleman N, Bron D, Toungouz M, Lagneaux L: Mesenchymal stromal cells use PGE2 to modulate activation and proliferation of lymphocyte subsets: Combined comparison of adipose tissue, Wharton's Jelly and bone marrow sources. Cell Immunol 2010, 264:171-179.

21. Tatara R, Ozaki K, Kikuchi Y, Hatanaka K, Oh I, Meguro A, Matsu H, Sato K, Ozawa K: Mesenchymal stromal cells inhibit Th17 but not regulatory T-cell differentiation. Cytotherapy 2011, 13:686-694.

22. Wang J, Wang G, Sun B, Li H, Mu L, Wang Q, Li G, Shi L, Jin L, Kostulas N: Interleukin-27 suppresses experimental autoimmune encephalomyelitis during bone marrow stromal cell treatment. J Autoimmun 2008 30:222-229

23. Zhao W, Wang Y, Wang D, Sun B, Wang G, Wang J, Kong Q, Wang Q, Peng H, Jin L, Li H: TGF-beta expression by allogeneic bone marrow stromal cells ameliorates diabetes in NOD mice through modulating the distribution of CD4+ T cell subsets. Cell Immunol 2008, 253:23-30.

24. Ren G, Zhao X, Zhang L, Zhang J, L'Huillier A, Ling W, Roberts Al, Le AD, Shi S, Shao C, Shi Y: Inflammatory cytokine-induced intercellular adhesion molecule- 1 and vascular cell adhesion molecule- 1 in mesenchymal stem cells are critical for immunosuppression. J Immuno/ 2010, 184:2321-2328.

25. Lim JH, Kim JS, Yoon IH, Shin JS, Nam HY, Yang SH, Kim SJ, Park CG: Immunomodulation of delayed-type hypersensitivity responses by mesenchymal stem cells is associated with bystander T cell apoptosis in the draining lymph node. J Immuno/ 2010, 185:4022-4029.

26. Ge W, Jiang J, Arp J, Liu W, Garcia B, Wang H: Regulatory T-cell generation and kidney allograft tolerance induced by mesenchymal stem cells associated with indoleamine 2,3-dioxygenase expression. Transplantation 2010, 90:1312-1320

27. Casiraghi F, Azzollini N, Cassis P, Imberti B, Morigi M, Cugini D, Cavinato RA, Todeschini M, Solini S, Sonzogni A, Perico N, Remuzzi G, Noris M: Pretransplant infusion of mesenchymal stem cells prolongs the survival of a semiallogeneic heart transplant through the generation of regulatory T cells. J Immuno/ 2008, 181:3933-3946.

28. Ankrum J, Karp JM: Mesenchymal stem cell therapy: two steps forward, one step back. Trends Mol Med 2010, 16:203-209.

29. Hermann-Kleiter N, Baier G: NFAT pulls the strings during CD4+ Thelper cell effector functions. Blood 2010, 115:2989-2997.

30. Aksu AE, Horibe E, Sacks J, Ikeguchi R, Breitinger J, Scozio M, Unadkat J, FeiliHariri M: Co-infusion of donor bone marrow with host mesenchymal stem cells treats GVHD and promotes vascularized skin allograft survival in rats.
Clin Immunol 2008, 127:348-358

31. Boumaza I, Srinivasan S, Witt WT, Feghali-Bostwick C, Dai Y, Garcia-Ocana A, Feili-Hariri M: Autologous bone marrow-derived rat mesenchymal stem cells promote PDX-1 and insulin expression in the islets, alter T cell cytokine pattern and preserve regulatory $T$ cells in the periphery and induce sustained normoglycemia. J Autoimmun 2009, 32:33-42.

32. Madec AM, Mallone R, Afonso G, Abou Mrad E, Mesnier A, Eljaafari A, Thivolet $\mathrm{C}$ : Mesenchymal stem cells protect NOD mice from diabetes by inducing regulatory T cells. Diabetologia 2009, 52:1391-1399.

33. Coffman RL: The origin of TH2 responses. Science 2010, 328:1116-1117.

34. Zhou H, Guo M, Bian C, Sun Z, Yang Z, Zeng Y, Ai H, Zhao RC: Efficacy of bone marrow-derived mesenchymal stem cells in the treatment of sclerodermatous chronic graft-versus-host disease: clinical report. Biol Blood Marrow Transplant 2010, 16:403-412.

35. Fiorina P, Jurewicz M, Augello A, Vergani A, Dada S, La Rosa S, Selig M, Godwin J, Law K, Placidi C, Smith RN, Capella C, Rodig S, Adra CN, Atkinson M, Sayegh $\mathrm{MH}, \mathrm{Abdi}$ : Immunomodulatory function of bone marrow-derived mesenchymal stem cells in experimental autoimmune type 1 diabetes. $\mathrm{J}$ Immuno/ 2009, 183:993-1004.

36. Awasthi A, Kuchroo VK: Th17 cells: from precursors to players in inflammation and infection. Int Immunol 2009, 21:489-498.

37. Ghannam S, Pene J, Torcy-Moquet G, Jorgensen C, Yssel H: Mesenchymal stem cells inhibit human Th17 cell differentiation and function and induce a T regulatory cell phenotype. J Immuno/ 2010, 185:302-312.

38. Zappia E, Casazza S, Pedemonte E, Benvenuto F, Bonanni I, Gerdoni E, Giunti D, Ceravolo A, Cazzanti F, Frassoni F, Mancardi G, Uccelli A: Mesenchymal stem cells ameliorate experimental autoimmune encephalomyelitis inducing T-cell anergy. Blood 2005, 106:1755-1761.

39. Rafei M, Campeau PM, Aguilar-Mahecha A, Buchanan M, Williams P, Birman E, Yuan S, Young YK, Boivin MN, Forner K, Basik M, Galipeau J: Mesenchymal stromal cells ameliorate experimental autoimmune encephalomyelitis by inhibiting CD4 Th17 T cells in a CC chemokine ligand 2-dependent manner. J Immuno/ 2009, 182:5994-6002.

40. Carrión F, Nova E, Luz P, Apablaza F, Figueroa F: Opposing effect of mesenchymal stem cells on Th1 and Th17 cell polarization according to the state of CD4(+) T cell activation. Immunol Lett 2011, 135:10-16.

41. Darlington PJ, Boivin MN, Renoux C, Francois M, Galipeau J, Freedman MS, Atkins HL, Cohen JA, Solchaga L, Bar-Or A: Reciprocal Th1 and Th17 regulation by mesenchymal stem cells: implication for multiple sclerosis. Ann Neurol 2010, 68:540-545.

42. Bouffi C, Bony C, Courties G, Jorgensen C, Noël D: IL-6-dependent PGE2 secretion by mesenchymal stem cells inhibits local inflammation in experimental arthritis. PLOS ONE 2010, 5:e14247.

43. Kong QF, Sun B, Wang GY, Zhai DX, Mu LL, Wang DD, Wang JH, Li R, Li HL: BM stromal cells ameliorate experimental autoimmune myasthenia gravis by altering the balance of Th cells through the secretion of IDO. Eur J Immunol 2009, 39:800-809.

44. Chen B, Hu J, Liao L, Sun Z, Han Q, Song Z, Zhao RC: Flk-1+ mesenchymal stem cells aggravate collagen-induced arthritis by up-regulating interleukin-6. Clin Exp Immunol 2010, 159:292-302.

45. Gonzalez-Rey E, Gonzalez MA, Varela N, O'Valle F, Hernandez-Cortes P, Rico L, Buscher D, Delgado M: Human adipose-derived mesenchymal stem cells reduce inflammatory and $T$ cell responses and induce regulatory $T$ cells in vitro in rheumatoid arthritis. Ann Rheum Dis 2010, 69:241-248.

46. Wang $Y$, Zhang $A$, Ye Z, Xie H, Zheng S: Bone marrow-derived mesenchymal stem cells inhibit acute rejection of rat liver allografts in association with regulatory T-cell expansion. Transplant Proc 2009, 41:4352-4356.

47. Afzali B, Mitchell P, Lechler Rl, John S, Lombardi G: Translational mini-review series on Th17 cells: induction of interleukin-17 production by regulatory T cells. Clin Exp Immuno/ 2009, 159:120-130.

48. Carrión F, Nova E, Ruiz C, Diaz F, Inostroza C, Rojo D, Monckeberg G, Figueroa FE: Autologous mesenchymal stem cell treatment increased T regulatory cells with no effect on disease activity in two systemic lupus erythematosus patients. Lupus 2010, 19:317-322

49. Maccario R, Podestà M, Moretta A, Cometa A, Comoli P, Montagna D, Daudt L, Ibatici A, Piaggio G, Pozzi S, Frassoni F, Locatelli F: Interaction of human mesenchymal stem cells with cells involved in alloantigen-specific immune response favors the differentiation of CD4+T-cell subsets expressing a regulatory/suppressive phenotype. Haematologica 2005, 90:516-525.

50. Rasmusson I, Ringden O, Sundberg B, Le Blanc K: Mesenchymal stem cells 
inhibit the formation of cytotoxic T lymphocytes, but not activated cytotoxic T lymphocytes or natural killer cells. Transplantation 2003, 76:1208-1213.

51. Prevosto C, Zancolli M, Canevali P, Zocchi MR, Poggi A: Generation of CD4+ or CD8+ regulatory T cells upon mesenchymal stem cell-lymphocyte interaction. Haematologica 2007, 92:881-888.

52. Patel SA, Meyer JR, Greco SJ, Corcoran KE, Bryan M, Rameshwar P: Mesenchymal stem cells protect breast cancer cells through regulatory T cells: role of mesenchymal stem cell-derived TGF-beta. J Immuno/ 2010, 184:5885-5894.
53. Tso GH, Law HK, Tu W, Chan GC, Lau YL: Phagocytosis of apoptotic cells modulates mesenchymal stem cells osteogenic differentiation to enhance IL-17 and RANKL expression on CD4+T cells. Stem Cells 2010, 28:939-954.

doi:10.1186/scrt75

Cite this article as: Duffy MM, et al:: Mesenchymal stem cell effects on T-cell effector pathways. Stem Cell Research \& Therapy 2011, 2:34. 